Pacific Journal of Mathematic 


\title{
A MORITA CONTEXT RELATED TO FINITE AUTOMORPHISM GROUPS OF RINGS
}

\author{
Miriam Cohen
}

\begin{abstract}
Let $R$ be a semiprime ring, $G$ a finite group of automorphisms of $R$ and $R^{G}$ the fixed ring. We investigate the associated Morita context $\left(R^{G}, R, R, R^{*} G\right)$, where $R^{*} G$ is the skew group ring. We then apply these results to two situations: (1) $G$ is $X$-outer (2) $R$ is $|G|$-torsion free.
\end{abstract}

o. Introduction and preliminaries. Let $R$ be a ring, $G$ a finite group of automorphisms of $R$ and $R^{G}=\left\{x \in R \mid x^{g}=x\right.$ for all $g \in G\}$. There has been considerable interest in the past years in studying connections between $R^{G}$ and $R$. The two major ways to approach the subject were the direct approach, and via the skew group ring $R * G$ which we denote by $S$. In this paper we investigate a third way which was used in the commutative case by Chase, Harrison and Rosenberg [5], and was suggested to us by S.A. Amitsur.

We consider an associated Morita context $\left[R^{G}, R, R, S\right]$ with $(x, y)=\sum_{g \in G}(x y)^{g}$ and $[x, y]=\sum_{g \in G} x y^{g^{-1}} g$, for all $x, y \in R$. This context incorporates all the relevant ingredients. The fixed ring is known to be nonzero in three major situations: (1) [11] $R$ is semiprime and $G$ is $X$-outer. (2) [4] $R$ is semiprime and $|G|$-torsion free (3) [11] $R$ has no nilpotent elements. Since in the third situation $t_{G}(x)=\sum_{g \in G} x^{g}$ might turn out to be 0 for all $x \in R$ [7], we apply the results of $\S 1$ only to the first two cases. It seems however plausible that by changing the context one could deal with the third situation by similar techniques.

Throughout the paper we assume that $R$ is a semiprime ring, an immediate consequence of which is that $[$,$] is a nondegenerate$ bilinear form. Another consequence is: if $\mathscr{N}(S)=0$ where $\mathscr{N}\left(^{*}\right)$ is the prime, Jacobson, locally nilpotent or nil radical, then $\mathscr{N}\left(R^{G}\right)=$ 0 [Lemma 1.2]. In $\S 1 \mathrm{~B}$ we investigate properties of the context when also $($,$) is assumed to be nondegenerate. We prove, among$ the rest, that $(R, R)$ is essential in $R^{G}$ and that when $R^{G}$ is semiprime then $R$ is Goldie (Artinian) iff $R^{G}$ is Goldie (Artinian); when $R$ is Artinian ther $($,$) is onto. [Theorem 1.6$ and Lemma 1.3.] Some of the results were proved by Montgomery [15]. Since $R$ is semiprime, it is a faithful $R^{\alpha}$-module, however, it need not be a faithful $S$-module. In $\S 1 \mathrm{C}$ we investigate the annihilator of $R$ in $S$, which turns out to be the annihilator (right or left) in $S$ of a two sided ideal of $S$, namely the ideal $[R, R]$. When $($,$) is non-$ 
degenerate and $R^{G}$ is prime then this annihilator is proved to be a minimal prime ideal of S. [Theorem 1.14.] We give equivalent conditions to faithfulness [Theorems 1.16, 1.17], and furthermore investigate the context when $R$ is assumed to be $S$-faithful. Among the rest, we show that $(R, R)$ and $[R, R]$ are essential in $R^{G}$ and $S$ respectively, that $($,$) is nondegenerate, and that d\left(_{R_{R}} R\right) \leqq$ $|G|^{2} d\left({ }_{R^{G}} R^{G}\right)$, where $d$ denotes the Goldie dimension. [Theorem 1.15.] We also show that $\mathscr{N}\left(R^{a}\right)=0$ iff $\mathscr{N}(S)=0$ with $\mathscr{N}\left({ }^{*}\right)$ as above, and that $R^{G}$ is primitive iff $S$ is primitive [Theorem 1.18]. When $R^{G}$ is assumed to be an Öre domain we prove, among the rest, that $S$ is a prime Goldie ring with $Q(S)=\left(Q\left(R^{G}\right)\right)_{n}$, where $n=d\left({ }_{S} S\right)$ [Theorem 1.19]. In $\S 1 \mathrm{D}$ we assume $S$ to be semiprime and prove, among the rest, that (, ) is nondegenerate and that $\hat{S}=S / \operatorname{Ann}_{S}(R)$ is Goldie iff $d\left({ }_{R} G R\right)<\infty$ and the singular ideal of $R^{G}$ is 0 , and then $R$ and $R^{G}$ are Goldie and $Q(\hat{S})=$ End $_{{ }^{G}}(Q(R))$. [Theorem 1.22.] In $\S 1 \mathrm{E}$ we consider the context under the assumption that $1 \in R$ and (, ) or [,] is onto, or equivalently when $R$ is a generator for ${ }_{R}^{G} \mathscr{C l}$ or ${ }_{S} \mathscr{C l}$ respectively. Since $R^{G} \cong \operatorname{End}_{S}(R)$, then [, ] being onto implies by the Morita theorem that $R$ is a finitely generated, projective $R^{G}$-module, $S \cong \operatorname{End}_{R^{G}}(R)$ and $R \bigotimes_{R^{G}} R \cong S$. If both ( , ) and [,] are onto then $R$ is called a $G$-Galois extension of $R^{G}$ [5], and then, among the rest, $R^{G}$ is Morita equivalent to $S$. We show that when $R$ is a semisimple Artinian ring and a faithful $S$-module then $R$ is a $G$-Galois extension of $R^{G}$ [Theorem 1.28]. In proving the results of this section we use extensively results of Amitsur [1].

In $\S 2$ we apply $\S 1$ to situations (1) and (2). By Fisher and Montgomery [8], $S$ is semiprime in both, and in case (1) $R$ is $S$ faithful. Moreover, if in case (1) $R$ is $G$-prime, primitive or $\mathscr{N}(R)=0$, with $\mathscr{N}\left({ }^{*}\right)$ as above, then the same is true for $S$. Hence if $R$ enjoys one of these properties so does $R^{G}$ [Theorem 2.3]. We thus give, among the rest, an affirmative answer to question 11 [10]. We also show that in case (1) $(R, R),[R, R],[R, R] \cap R$ and $[R, R] \cap R^{G}$ are essential ideals of $R^{G}, S, R$ and $R^{G}$ respectively; that $S$ is Goldie iff $R^{G}$ is Goldie iff $R$ is Goldie and that if $R^{G}$ is an Öre domain then $Q(S) \cong\left(Q\left(R^{G}\right)\right)_{n}$ [Theorems 2.2, 2.3]. If $R$ is semisimple Artinian or if $R^{G}$ is simple then $R$ is a $G$-Galois extension over $R^{G}$ [Theorems 2.4, 2.5]. This extends a theorem of Azumaya and Nakayama [3]. When $R$ is simple, [ , ] is onto hence results of $[14,19,20]$ follow directly.

Let us define some terms, for a complete survey see [18]. Let $R$ be a semiprime ring, (that is, without nontrivial nilpotent ideals), $\mathscr{F}$ the filter of essential ideals of $R$, and $R_{\mathscr{F}}$ the (left) quotient ring of $R$ with respect to $\mathscr{F}$. Then $R \subset R_{\mathscr{F}}$, denote by $C=$ center of $R_{\mathscr{F}}$. For any $g \in \operatorname{Aut}(R), g$ has a unique extension to $R_{\mathscr{F}}$. Define 
$\phi_{g}=\left\{x \in R_{\mathscr{F}} \mid x r=r^{g} x\right.$, for all $\left.x \in R\right\}$. We say that $g$ is $X$-inner if $\dot{\phi}_{g} \neq 0$. This is a generalization of the usual notion of inner. By [12] $\phi_{g}=C x_{g}$, a cyclic $C$-module. If $\phi_{g}=0$, then $g$ is called $X$-outer. For any group $G \subset \operatorname{Aut}(R)$, let $G_{\text {inn }}$ be the set of $X$-inner automorphisms in $G$. If $G_{\mathrm{inn}}=\{1\}$, we say that $G$ is $X$-outer. The algebra of the group $B=\sum_{g \in G} \phi_{g}$.

A ring related to both $R$ and $G$ is the skew group ring $R * G$ which will be denoted henceforth by $S$. The ring $S$ is defined to be $\sum_{g \in G} \oplus R_{g}$ with addition given componentwise and multiplication given as follows: if $x, y \in R$ and $g, h \in G$ then $(x g)(y h)=x y^{g^{-1}} g h$. Note that $y g=g y^{g}$. We define $R_{\mathscr{F}} * G$ similarly. If $s=\sum r_{g} g \in S$, then the support of $s$ is the set $\operatorname{supp}(s)=\left\{g \in G \mid r_{g} \neq 0\right\}$. Fisher and Montgomery [8] have proved an important connection between $G_{\text {inn }}$ and ideals of $S$. They showed:

THEOREM 0.1 [8]. Let $R$ be a semiprime ring, and $G$ a group $\subset \operatorname{Aut}(R)$ (not necessarily finite). Let $I$ be a nonzero ideal of $S$, and let $x=\sum r_{g} g$ be an element of $I$ of minimal support. Then $\operatorname{supp}(x) \subset G_{\text {in }}$, and $x=a \sum x_{g} g$, where $a \in R$ and $x_{g} \in \phi_{g}$. In particular, if $G$ is $X$-outer then every nonzero ideal of $S$ intersects $R$ nontrivially.

They used this result to prove the important:

THEOREM 0.2 [8]. Let $R$ be a semiprime ring and $G$ a finite group of automorphisms of $R$. If either $R$ has no $|G|$-torsion or $G$ is $X$-outer then $S$ is semiprime.

The proof of Theorem 0.1 which essentially appeared in [15], depended on a notion of linear independence introduced in [11]. A different and more elementary approach was used in [13, Lemma 1.5] for prime rings and can be extended to semiprime rings by the methods of [7, Lemma 1.5].

A Morita context [1] is a set $M=(R, V, W, S)$ and two maps (, ) and [, ]; where $R$ and $S$ are rings, $V$ is an $R-S$ bimodule and $W$ is an $S-R$ bimodule. The map (, ): $V \otimes_{S} W \rightarrow R$ is an $R-R$ bilinear map, and [, ]: $W \otimes_{R} V \rightarrow S$ is $S-S$ bilinear. Furthermore, these maps satisfy the following associativity conditions: $1_{V} \otimes[]=,(,) \otimes 1_{V}$ and $[,] \otimes 1=1 \otimes($,$) . That is,$ for all $v, v^{\prime} \in V$ and $w, w^{\prime} \in W$ we have: $v \cdot\left[w, v^{\prime}\right]=(v, w) \cdot v^{\prime}$ and $[w, v] \cdot w^{\prime}=w \cdot\left(v, w^{\prime}\right)$

We shall also use the following known notions and symbols: Let ${ }_{R} \mathscr{C}$ denote the category of left $R$-modules. Let ${ }_{R} M$ be a left $R$-module, then $d\left({ }_{R} M\right)$ - the Goldie dimension of ${ }_{R} M$ as a left $R$ - 
module is the supremum of the length of direct sums of submodules of $M$. In particular, ${ }_{R} M$ will be said to be uniform if $d\left({ }_{R} M\right)=1$. A submodule ${ }_{R} E \subset_{R} M$ is essential (large) in ${ }_{R} M$ if $E \cap U \neq 0$ for every nonzero submodule $U$ of $M$. The (left) singular submodule $Z\left({ }_{R} M\right)=\{m \in M \mid E m=0$, for some essential left ideal $E$ of $R\}$. By $\operatorname{Ann}_{R}(M)$ we denote $\{r \in R \mid r M=0\}$, it is an ideal of $R$. If $\operatorname{Ann}_{R} M=$ 0 then $M$ is called a faithful $R$-module. $M$ is called torsion-free if $r m=0$ implies $r=0$ or $m=0$. For any nonempty subset $A$ of $R$ we define $r_{R}(A)=\{r \in R \mid A r=0\}$ and by $\ell_{R}(A)=\{r \in R \mid r A=0\}$. Let $R$ be a semiprime Goldie ring. Denote by $Q\left({ }_{R} R\right)$ or in short $Q(R)$ its classical ring of quotients, and for each left $R$-module ${ }_{R} M$ denote by $Q\left({ }_{R} M\right)=M \Theta_{R} Q(R)$, its module of fractions. If $Z\left({ }_{R} M\right)=0$ then by [21, Cor. 2.8], $M \otimes_{R} Q\left({ }_{R} R\right)$ is an injective envelope of ${ }_{R} M$. A ring $R$ is called prime if whenever $A, B$ are ideals of $R$ with $A B=$ 0 , then $A=0$ or $B=0$. It is called $G$-prime (with $G \subset \operatorname{Aut}(R)$ ) if the same holds for $A, B$ which are $G$-invariant ideals. It is easy to see that if $S$ is prime then $R$ is $G$-prime, which was shown [13] to imply that $R$ is semiprime.

1A. The associated Morita context. Throughout, let $R$ be a semiprime ring and $G$ a finite group of automorphisms of $R$. Let $S=R^{*} G$, then $R$ may be viewed as a right or left $S$-module as follows: for any $s=\sum r_{g} g \in S$ and $r \in R$, define: $s \cdot r=\left(\sum r_{g} g\right) \cdot r=$ $\sum r_{g} r^{g^{-1}}$ and $r \cdot s=\sum\left(r r_{g}\right)^{g}$. This definition extends the product in $R$. Note that the $S$-submodules of ${ }_{S} R\left(R_{S}\right)$ are the $G$-invariant left (right) ideals of $R$. In order to differentiate between the product of $r$ and $s$ as elements of $S$ and the above module action, we shall throughout indicate the module action by a dot. $R$ is obviously an $R^{G}$-bimodule, and in fact $R$ is an $R^{G}-S$ and an $S-R^{G}$ bimodule. In order to differentiate between the two let $V={ }_{R^{G}} R_{S}$ and $W=$ ${ }_{s} R_{R_{G}}$. Let $t=\sum_{g \in G} g$ be the formal sum, then for any $r \in R, t_{G}(r)$ is formally $r \cdot t$ (or $t \cdot r$ ). If $1 \in R$ then $t$ is an element of $S$. Let us record some easily verified facts:

(1) $g t=t g=t$ for all $g \in G$. Hence $S t=R t$ and $t S=t R$,

(2) if $s \in S$ and $r \in R$ then $s r t=(s \cdot r) t$ and $t r s=t(r \cdot s)$,

(3) for any $r \in R$ and $g \in G, r \cdot t=t \cdot r=r^{g} \cdot t$,

(4) for any $x \in R^{a}, x t=t x$,

(5) if $r, r^{\prime} \in R$ then $\left(r r^{\prime}\right) \cdot t=r \cdot\left(r^{\prime} t\right)=(t r) \cdot r^{\prime}$.

Now let us define:

$():, V \otimes W \rightarrow R^{G}$ by: $(v, w)=t_{G}(v w)=v w \cdot t$ for any $v \in V$ and $w \in W$. Obviously $($,$) is an R^{G}$-bimodule homomorphism and furthermore $(v \cdot s, w)=(v, s \cdot w)$ for any $v \in V, w \in W$ and $s \in S$. For, $(v \cdot s, w)=((v \cdot s) w) \cdot t$, however $s, w \in S$, hence $(v \cdot s) w=v \cdot s w$, thus $((v \cdot s) w) \cdot t=v \cdot(s w t)$. By (2) $s w t=(s \cdot w) t$, thus $v \cdot s w t=v \cdot((s \cdot w) t)$, 
which by (5) equals $(v(s \cdot w)) \cdot t=(v, s \cdot w)$. Next define [ , ]: $W \boldsymbol{\bigotimes}_{R^{G}} V \rightarrow$ $S$ by: $[w, v]=w \sum_{g \in G} v^{g^{-1}} g=w t v$. The map [,] is an $S$-bimodule homomorphism. For, if $s \in S$, then $s[w, v]=s(w t v)=(s w t) v$ which by (2) equals $((s \cdot w) t) v=[s \cdot w, v]$. Similarly, $[w, v] s=[w, v \cdot s]$. Now let $x \in R^{G}$, then by (4) $[w x, v]=[w, x v]$. Finally, we show that these maps satisfy the associativity conditions: $v \cdot\left[w, v^{\prime}\right]=(v, w) v^{\prime}$ and $[w, v] \cdot w^{\prime}=w\left(v, w^{\prime}\right)$. Well, $v \cdot\left[w, v^{\prime}\right]=v \cdot\left(w t v^{\prime}\right)=(v \cdot w t) v^{\prime}$ which by (5) equals $(v, w) v^{\prime}$. The second condition is proved similarly.

Note that for any $w \in W, v \in V,[w, V]([W, v])$ is a right (resp. left) ideal of $S$ and $(v, W)((V, w))$ is a right (resp. left) ideal of $R^{G}$. In particular, $[W, V]$ is an ideal of $S$ and $(V, W)$ is an ideal of $R^{G}$.

LEMMA 1.1. If $w \in W$ then $w^{\perp}=\{v \in V \mid[w, v]=0\}$ is a Ginvariant right ideal of $R$ contained in $r_{R}(w)$. Similarly, $v^{\perp}=$ $\{w \in W \mid[w, v]=0\}$ is a G-invariant left ideal of $R$ contained in $\ell_{R}(v)$.

Proof. Let $v \in W^{\perp}$ and $g \in G$ then $\left[w, v^{g}\right]=[w, v \cdot g]=[w, v] g=0$. Hence $w^{\perp}$ is $G$-invariant, the rest is obvious.

A bilinear form $f: A \times B \rightarrow C$ where $A, B, C$ are additive groups is called nondegenerate if for any $0 \neq a \in A$ and $0 \neq b \in B, f(a, B) \neq 0$ and $f(A, b) \neq 0$. A consequence of the previous lemma is that [,] is always nondegenerate when $R$ is semiprime.

LEMMA 1.2. Let $R$ be a semiprime ring. Then:

(a) $[$,$] is nondegenerate.$

(b) Let $\mathscr{N}\left(^{*}\right)$ denote one of the following radicals: lower, locally nilpotent, Jacobson or nil. Then $\mathscr{N}(S)=0$ implies $\mathscr{N}\left(R^{G}\right)=0$.

(c) If $I$ is a minimal left ideal of $S$ then $V \cdot I$ is a simple $R^{G}$-module.

Proof. (a) Since $R$ is semiprime, $r_{R}(w) \neq R$ and $\ell_{R}(v) \neq R$, hence the result follows from Lemma 1.1.

(b) By [1, Corollary 23], $\left[w, \mathscr{N}\left(R^{G}\right) V\right] \subset \mathscr{N}(S)$.

The result now follows from part (a) and the semiprimeness of $R$.

(c) If $V \cdot I=0$ then we are done. Otherwise, assume that $0 \neq J \subset V \cdot I$, where $J$ is a left $R^{G}$-submodule of $R$. Then by part (a), $[W, J] \neq 0$; thus $0 \neq[W, J] \subset[W, V \cdot I]=[W, V] I \subset I$. By minimality of $I,[W, J]=I$. But then, $V \cdot[W, J]=V \cdot I$, however, $V$. $[W, J]=(V, W) J \subset J$. Hence we have: $J \subset V \cdot I=(V, W) J \subset J$, which implies $J=V \cdot I$. We have shown that $V \cdot I$ is a simple $R^{a_{-}}$ module. 
1B. (,$)$ is nondegenerate. In the following we prove some consequences of nondegeneracy of $($,$) . Most of them are noted in$ [1] and [15].

LEMMA 1.3. Let $R$ be a semiprime ring and assume (, ) is nondegenerate then: $v=0$.

(a) $[W, V] \cdot w=0$ implies $w=0$ and $v \cdot[W, V]=0$ implies

(b) $\ell_{R}(V, W)=r_{R}(V, W)=0 . \quad$ In particular, $(V, W)$ is an essential ideal of $R^{G}$.

(c) If $A \subset R^{G} \quad$ and $\quad r_{R^{G}}(A)=0 \quad\left(\ell_{R^{G}}(A)=0\right)$ then $\quad r_{R}(A)=0$ $\left(\ell_{R}(A)=0\right)$.

(d) If $E$ is an essential left ideal of $R$, or an essential $R^{G}$ submodule of $R$, then $(V, E)$ is an essential left ideal of $R^{G}$.

(e) $\quad d\left({ }_{R} G R^{G}\right)=d\left({ }_{S} R\right) \leqq d\left({ }_{R} R\right) \leqq|G| d\left(_{{ }_{R} G} R^{G}\right)$.

Proof. (a) By [1, Corollary 3].

(b) Assume $r(V, W)=0$ for some $r \in R$. Then $((V, r) V, W)=$ $(V \cdot[r, V], W)=(V,[r, V] \cdot W)=(V, r(V, W))=0$. Thus by nondegeneracy of $($,$) we have (V, r) V=0$ which by semiprimeness of $R$ and nondegeneracy of ( , ) implies $r=0$. Similarly, $r_{R}(V, W)=0$.

(c) For any $A \subset R^{G},\left(V, \ell_{R}(A)\right) \subset \ell_{R^{G}}(A)$ and $\left(r_{R}(A), W\right) \subset r_{R^{G}}(A)$. Hence if $r_{R^{G}}(A)=0$, then by nondegeneracy of $(),, r_{R}(A)=0$. Similarly $\ell_{R} G(A)=0$.

(d) Assume $E$ is an essential left ideal of $R$ and let $A$ be a left ideal of $R^{G}$, then $E \cap R A \neq 0$. Thus choose $r \in R$ and $a \in A$ such that $0 \neq r a \in E$. But then $0 \neq(V, r a)=(V, r) a \subset A$ hence $(V, E) \cap A \neq 0$. The rest follows similarly.

(e) $d\left({ }_{R}{ }_{G} R^{G}\right)=d\left({ }_{S} R\right)$ by [1, Corollary 3].

The proof that $d\left({ }_{R} R\right) \leqq|G| d\left({ }_{R} G R^{G}\right)$ is the same as in [9] since nondegeneracy of $($,$) implies that any nontrivial G$-invariant right (left) ideal of $R$ intersects $R^{G}$ nontrivially.

CoROllaRy 1.4. If $R^{G}$ is an integral domain, $R$ is semiprime and $($,$) is nondegenerate then [w, v]=0$ implies $w=0$ or $v=0$.

Proof. Assume $[w, v]=0$, for $w \in W$ and $v \in V$. Then $0=[w, v]$. $W=w(v, W)$. If $v \neq 0$ then by nondegeneracy, $0 \neq(v, W) \subset R^{c}$. Since $\ell_{R^{G}}((v, W))=0$ we deduce from Lemma 1.3c that $w=0$.

CoROLLARY 1.5. Let $R$ be semiprime, (, ) nondegenerate and $Z\left({ }_{R^{G}} R^{G}\right)=0$. Then $Z\left(_{R} G\right)=0$ and $Z\left({ }_{R} R\right)=0$.

Proof. Let $E$ be an essential left $R^{G}$ submodule of $R$ then by 
Lemma 1.3.d $(V, E) \cap E$ is an essential left ideal of $R^{G}$. Since $r_{{ }_{R} G}((V, E) \cap E)=0$, Lemma 1.3.c implies that $r_{R}((V, E) \cap E)=0$ hence $r_{R}(E)=0$, and thus $Z\left(_{R}{ }^{G} R\right)=0$. Now, if $E$ is an essential left ideal of $R$ then $\bigcap_{g \in G} E^{g} \subset E$ is essential $G$-invariant left ideal of $R$, thus $\left(V, \cap E^{g}\right) \subset \cap E^{g} \subset E$. By Lemma 1.3.d $\left(V, \cap E^{g}\right)$ is essential in $R^{G}$, hence as above $r_{R}(E)=0$.

THEOREM 1.6 [18]. Let $R$ and $R^{G}$ be semiprime rings and assume $($,$) is nondegenerate. Then:$

(a) $R^{G}$ is Goldie if and only if $R$ Goldie, and then $Q\left({ }_{R} R\right)=$ $Q\left({ }_{R_{i}} R\right)$.

(b) $R^{a}$ is semisimple Artinian if and only if $R$ is semisimple Artinian, and then (, ) is onto.

(a) Is proved as in [18] using Lemma 1.3, Corollary 1.5 and an argument as in [6].

(b) A criterion for being semisimple Artinian is having no nontrivial essential left (right) ideals. Hence (b) is proved using Lemma 1.3.b,c and part (a). Also, by Lemma 1.3.b $(V, W)$ is essential in $R^{G}$ hence equals it. That is, $($,$) is onto.$

1C. $R$ is a faithful $S$-module. Since we deal with a semiprime ring $R, V$ and $W$ are faithful $R^{a}$-modules. However, they need not be faithful $S$-modules (Example 2.1). We shall consider in the following lemmas $\mathrm{Ann}_{S} V$ and $\mathrm{Ann}_{S} W$, and then proceed to the case in which they are 0 . Since these annihilators are ideals in $S$, Theorems 0.1 and 0.2 are applicable. First an easily verified:

REMARK 1.7. If $x_{g} \in \phi_{g}$ and $v \in V(w \in W)$ then $v \cdot x_{g} g=x_{g} v$ $\left(x_{g} g \cdot w=w x_{g}\right)$.

Let $A$ be an ideal of $S$, denote by $\min (A)$ the set of elements of $A$ of minimal support; $\min (A)$ is obviously an $R$-submodule of $S$.

Proposition 1.8. Let $R$ be a semiprime ring. Then $\min \left(\operatorname{Ann}_{S} W\right)=$ $\min \left(\mathrm{Ann}_{S} V\right)$.

Proof. Let $s \in \min \left(\mathrm{Ann}_{S} V\right)$, then by Theorem 0.2, $s=a \sum x_{g} g$, with $a \in R$ and $x_{g} \in \phi_{g}$. Let $v \in V$, then by Remark $1.7,0=v \cdot s=$ $v \cdot\left(a \Sigma x_{g} g\right)=\Sigma x_{g} v a$. Since $v$ was arbitrary, we have $\left(\Sigma x_{g}\right) R a=0$. By [16] there exists an $I \in \mathscr{F}$ such that $\left(\Sigma x_{g}\right) I \subset R$ and $I\left(\Sigma x_{g}\right) \subset R$. Hence $a R \Sigma x_{g} I$ is a right ideal of $R$ of square 0 , thus $a R \Sigma x_{g}=0$. By Remark 1.7 this implies that $s \cdot W=0$. Hence $\min \left(\operatorname{Ann}_{s} V\right) \subset$ $\mathrm{Ann}_{S} W$. Similarly, $\min \left(\mathrm{Ann}_{S} W\right) \subset \mathrm{Ann}_{S} V$. Hence $\min \left(\mathrm{Ann}_{S} V\right)=$ $\min \left(\operatorname{Ann}_{S} W\right)$. 
CoROLlaRY 1.9. Let $R$ be a semiprime ring. Then $\operatorname{Ann}_{S} V=0$ if and only if $\mathrm{Ann}_{s} W=0$.

Since $[$,$] is nondegenerate, it is easy to verify$

LEMMA 1.10. Let $R$ be a semiprime ring. Then $\operatorname{Ann}_{S} W=$ $\iota_{S}[W, V]$ and $\mathrm{Ann}_{S} V=r_{s}[W, V]$.

Corollary 1.11. Assume $S$ is a semiprime ring, and [ $W, V]$ is an essential right (left) ideal of $S$ then ${ }_{s} W$ is a faithful $S$ module.

Let us note in passing a result which can be easily deduced from [4].

LEMMA 1.12. Let $R$ be a semiprime ring. If $s=\Sigma r_{g} g \in \mathrm{Ann}_{s} W$ then $\Sigma r_{g}=0$.

The following appears in the proof of [1, Theorem 20] for general Morita contexts. It gives a correspondence between prime ideals of $R^{G}$ and $S$.

LEMMA 1.13. Let $R$ be a semiprime ring. Let $P$ be a prime ideal of $R^{G}$ then $P_{S}=\{s \in S \mid[W, V] s[W, V] \subset[W, P V]\}$ is a prime ideal of $S$. Symmetrically, if $Q$ is a prime ideal of $S$ then

$$
Q_{R^{G}}=\left\{x \in R^{G} \mid(V, W) x(V, W) \subset(V, Q \cdot W)\right\} \text { is a prime ideal of } R^{G} \text {. }
$$

THEOREM 1.14. Let $R$ be a semiprime ring and assume (, ) is nondegenerate. If $R^{G}$ is a prime ring then $\operatorname{Ann}_{S}(W)$ is a minimal prime ideal of $S$.

Proof. Since $R^{G}$ is a prime ring, $P=0$ is a prime ideal of $R^{G}$. Hence by Lemma $1.13 P_{S}=\{s \in S \mid[W, V] s[W, V]=0\}$ is a prime ideal of $S$. We show that $P_{S}=\operatorname{Ann}_{S}(W)$ and then since $A_{n}(W) \cap$ $R=0$ it is a minimal prime by [13]. Well, obviously $\operatorname{Ann}_{S}(W) \subset P_{S}$. Conversely, let $s \in P_{S}$, then $[W, V \cdot[s \cdot W, V]]=0$. By nondegeneracy of $[$,$] , we get 0=V \cdot[s \cdot W, V]=(V, s \cdot W) V$, implying $(V, s \cdot W)=$ 0 . Now by nondegeneracy of ( , ) it follows that $s \in \operatorname{Ann}_{s}(W)$. Hence $P_{S}=\operatorname{Ann}_{S}(W)$ and the result is proved.

In the following we prove several consequences of $S$-faithfulness. We show, among the rest, that the Morita context has nice properties and that $[W, V]$ and $(V, W)$ are essential. But first a definition. A finite set of elements $\left\{x_{i}\right\} \subset R_{\mathscr{T}}$ is called $C$-weakly inde- 
pendent if $\Sigma c_{i} x_{i}=0$, where $c_{i} \in C$, implies $c_{i} x_{i}=0$ for all $i$.

THEOREM 1.15. Let $R$ be a semiprime ring and assume ${ }_{S} W$ (or $\left.V_{S}\right)$ is S-faithful, then:

(a) $r_{S}[W, V]=\iota_{S}[W, V]=0$, in particular, $[W, V]$ is an essential right (left) ideal of $S$.

(b) [, ] and ( , ) are nondegenerate.

(c) $(V, W)$ is an essential right (left) ideal of $R^{G}$.

(d) To each $g \in G_{\mathrm{inn}}$ associate an $x_{g}$ such that $\dot{\phi}_{g}=C x_{g}$, then the set $\left\{x_{g}\right\}_{g \in G_{\text {in }}}$ is weakly C-independent.

(e) $(V, s \cdot W)=0$ implies $s=0$.

(f) $d\left({ }_{R^{G}} R\right)=d\left({ }_{S} S\right)=|G| d\left({ }_{R} R\right) \leqq|G|^{2} d\left(_{{ }_{R} G} R^{G}\right)$.

Proof. (a) is a consequence of Corollary 1.9 and Lemma 1.10.

(b) $[$,$] is nondegenerate by Corollary 1.2. Now assume$ $(v, W)=0$ for some $v \in V$. Then, $0=W(v, W)=[W, v] \cdot W . \quad$ Вy faithfulness and nondegeneracy of [,] we deduce that $v=0$.

(c) Follows from (b) and Lemma 1.3.b.

(d) If $\Sigma c_{g} x_{g}=0$ with $g \in C$, then by Remark 1.7 and since $c_{g} \in C$, we have $\left(\Sigma c_{g} x_{g} g\right) W=W \Sigma c_{g} x_{g}=0$. Hence, $\Sigma c_{g} x_{g} g=0$, but then $c_{g} x_{g}=0$ for each $g$.

(e) Follows from (b) and $S$-faithfulness.

(f) By [1, Theorem 2] $d\left({ }_{R} G\right)=d\left({ }_{S} S\right)$.

Since $S$ is a direct sum of $|G|$ copies of $R, d\left({ }_{R} S\right)=|G| d\left({ }_{R} R\right)$. However, $d\left({ }_{S} S\right) \leqq d\left({ }_{R} S\right)$ and $d\left({ }_{R} R\right) \leqq|G| d\left(_{R} R^{G}\right)$ by (b) and Lemma 1.3.e. Hence $d\left({ }_{S} S\right) \leqq|G|^{2} d\left(_{{ }_{L} G} R^{G}\right)$.

The next two theorems constitute a generalization of $[16$, Theorem 7] from domains to prime rings.

THEOREM 1.16. Let $R$ be a semiprime ring. Then the following are equivalent:

(1) $S$ is a prime ring.

(2) ${ }_{S} W$ is a faithful $S$-module and $R^{G}$ is a prime ring.

Proof. $(1) \Rightarrow(2)$ since $[W, V]$ is an ideal of $S$, a prime ring, $\mathrm{Ann}_{S}[W, V]=0$. Hence by Lemma $1.10{ }_{S} W$ is $S$-faithful. $R^{G}$ is prime for by Lemma 1.13. $Q_{R^{G}}=\left\{x \in R^{G} \mid(V, W) x(V, W)=0\right\}$ is a prime ideal of $R^{G}$. However, since ${ }_{s} W$ is a faithful $S$-module Theorem 1.15.c implies that $Q_{R^{G}}=0$.

$(2) \Rightarrow(1)$ by Theorem 1.15.b (, ) is nondegenerate hence by Theorem $1.14 \mathrm{Ann}_{S} W=0$ is a prime ideal of $S$.

If $R$ is assumed to be a prime ring we can show another equivalence which is implicit in the proof of [16]. 
THEOREM 1.17. Let $R$ be a prime ring, then the following are equivalent:

(1) $W$ is a faithful S-module.

(2) $\operatorname{dim}_{C} B=\left|G_{\mathrm{inn}}\right|$.

Proof. (1) $\Longrightarrow(2)$ follows from Theorem 1.15.d and the fact that $C$ is a field.

$(2) \Rightarrow(1)$ if $s \in \min \left(\mathrm{Ann}_{S} W\right)$ then $s=a \Sigma x_{g} g$, where $a \in R$. As in the proof of Proposition 1.8 this implies that $a R I \Sigma x_{g}=0$ for some $I \in \mathscr{F}$ such that $I \Sigma x_{g} \subset R$. By primeness of $R$ this implies that $a=0$ or $\Sigma x_{g}=0$. Since $B$ is a vector space over $C$ of dimension $\left|G_{1 \mathrm{~m}}\right|$ and since $\left\{x_{g} \mid g \in G_{\mathrm{inn}}\right\}$ generate $B$ over $C$ we have $\Sigma x_{g} \neq 0$. Hence $a=0$, which implies $s=0$. We have shown that $\mathrm{Ann}_{S} W=0$.

As a consequence of Theorem 1.15 and results from [1] we get the following correspondences between $S$ and $R^{G}$.

THEOREM 1.18. Let $R$ be a semiprime ring and assume ${ }_{s} W$ is a faithful S-module, then:

(a) $\mathscr{N}(S)=0$ if and only if $\mathscr{N}\left(R^{(i)}=0\right.$, where $\mathscr{N}\left({ }^{*}\right)$ is one of the following radicals: lower, locally nilpotent, Jacobson or nil.

(b) $R^{G}$ is primitive if and only if $S$ is primitive.

Proof. (a) By [1, Theorem 20 and Corollary 23] $(V, \mathscr{V}(S) \cdot W) \subset$ $\mathscr{N}\left(R^{G}\right)$ and $\left[W, \mathscr{N}\left(R^{G}\right) V\right] \subset \mathscr{N}(S)$. By Theorem 1.15 [, ] and (, ) are nondegenerate hence the result follows from $R^{G}$ and $S$ faithfulness.

(b) By Theorem 1.15.e, $(V, s \cdot W)=0$ implies $s=0$. Hence by [1, Theorem 27] if $R^{G}$ is primitive then so is $S$. Symmetrically, since $[W, x V]=0$, (where $x \in R^{G}$ ) implies $x=0$, we have, as above, the reverse implication.

When $R^{a}$ is an Öre domain and ${ }_{S} W$ is a faithful $S$-module then $S$ has a very specific characterization.

THEOREM 1.19. Let $R$ be a semiprime ring, ${ }_{s} W$ a faithful $S$ module and $R^{G}$ an Öre domain, then:

(a) $S$ is dense (in the sense of [1]) in $\operatorname{End}_{k_{G}}(R)$.

(b) $S$ is a prime Goldie ring, with $d\left({ }_{S} S\right)=n=d\left({ }_{R i} R\right) \leqq|G|^{2}$.

(c) $Q(S)=\operatorname{End}_{H^{G}}(Q(R))=\left(Q\left(R^{f}\right)\right)_{n}$.

(d) $d\left({ }_{s} R\right)=1$.

(e) ${ }_{{ }_{i} G} R$ and $R_{l_{i} G}$ are torsion-free.

Proof. Let us apply [1, Theorem 4] to the Morita context $\left(S, W, V, R^{(i)}\right.$ ) with change of roles of the maps $($,$) and [ , ]. By$ Theorem 1.15.b ( , ) is nondegenerate hence by Lemma 1.3.e $d\left({ }_{s} W\right)=$ 
$d\left({ }_{R} G R^{G}\right)=1$, hence (d). Furthermore, the product [, ] satisfies by Corollary 1.4 the property: $[w, v]=0$ iff $w=0$ or $v=0$; and by Lemma 1.3.a the property: $[w, V] \cdot w=0$ iff $w=0$. We have shown that condition 2 of [1, Theorem 4] is satisfied in our context hence:

(a) follows from [1, Theorem 4 (3)].

(b) follows from Theorem 1.16 and Theorem 1.15.f.

(c) follows from [1, Theorem 10.c.3] and Theorem 1.23.

(d) follows from Theorem 1.3.c or [1, Lemma 6].

We shall return to further properties of $S$-faithfulness in the following sections.

1D. $S$ is semiprime. When $S$ is assumed to be semiprime, $R$ is easily seen to be semiprime. In both applications of $\S 2, S$ is in fact semiprime, as noted in $\S 0$. In the following we prove consequences of this assumption, some of which were proved in [15]. Next we define prime (semiprime) Morita contexts [22]. A Morita context is said to be prime (semiprime) if for every $0 \neq v \in V$, $0 \neq v_{1} \in V,(v, W) v_{1} \neq 0((v, W) v \neq 0)$.

THEOREM 1.21. If $S$ is a semiprime ring, then:

(a) [, ] and (, ) are nondegenerate.

(b) $R$ and $R^{G}$ are semiprime.

(c) $R^{G}$ is Goldie if and only if $R$ is Goldie and then $Q(R)=$ $Q\left({ }_{R^{G}} R\right)$.

(d) $R^{G}$ is Artinian if and only if $R$ is Artinian and then (, ) is onto.

(e) $(V, W)$ is an essential ideal of $R^{a}$.

(f) $\left(R^{G}, V, W, S\right)$ is a semiprime Morita context.

(g) $\hat{S}=S / \mathrm{Ann}_{S} V$ is a semiprime ring.

Proof. (a) [, ] is nondegenerate since $R$ is semiprime. Now, if $(v, W)=0$ then $[W, v][W, v]=[W, v \cdot[W, v]]=[W,(v, W) v]=0$. Hence by semiprimeness of $S,[W, v]=0$ which by nondegeneracy of [ , ] implies $v=0$.

(b) Follows from Lemma 1.2.b.

(c), (d) follows from (a) and Theorem 1.6.

(e) Follows from (a) and Lemma 1.3.b.

(f) If $(v, W) v=0$ for some $v \in V$. Then, $0=[W,(v, W) v]=$ $[W(v, W), v]=[[W, v] \cdot W, v]=[W, v]^{2}$. Since $[W, v]$ is a left ideal of $S$ this implies $[W, v]=0$, hence $v=0$.

(g) Since $[W, V]$ is an ideal in a semiprime ring $S$, it is routine that $S / \mathrm{Ann}_{S}[W, V]$ is semiprime. Since $\mathrm{Ann}_{S} V=\mathrm{Ann}_{S}[W, V]$, (g) is proved. 
The following two theorems are consequences of Theorem 1.21 and [22, Theorem 3.5] and form a generalization of Theorem 1.19.

THEOREM 1.22. Let $R$ be a ring. If $S$ is semiprime then $\hat{S}=$ $S / \mathrm{Ann}_{S} R$ is Goldie if and only if $d\left({ }_{R} G\right)<\infty$ and $Z\left({ }_{R} G R^{G}\right)=0$. And then $R$ and $R^{G}$ are semiprime Goldie and $Q(\hat{S}) \cong \operatorname{End}_{R^{G}}(Q(R))$.

Proof. By Theorem 1.21.a (, ) is nondegenerate hence by Lemma 1.3.b $(V, W) v \neq 0$ if $0 \neq v \in V$. Furthermore, by Corollary $1.5 Z\left(_{R_{G}} R^{G}\right)=0$ implies $Z\left(_{R} R\right)=0$. The context is semiprime by Theorem 1.21.f hence if $d\left({ }_{R} G\right)<\infty$ and $Z\left({ }_{R} G R^{G}\right)=0$ then by [22] $\hat{S}$ is Goldie. Conversely, if $\hat{S}$ is Goldie, then by Theorem 1.21.g it is semiprime Goldie. Since $R^{G}$ is isomorphic to a subring of $\hat{S}$ it inherits the chain condition on annihilators. Now by [22] $d\left({ }_{R} G R\right)<$ $\infty$, hence $d\left({ }_{R G} R^{G}\right)<\infty$. Thus, since $R^{G}$ is semiprime it is semiprime Goldie implying $Z\left({ }_{R} G R^{G}\right)=0$. By Theorem 1.6.a $R$ is semiprime Goldie and $Q(R)=Q\left(_{R^{G}} R\right)$ and by $\S 0 . \quad Q\left(_{R} G\right)$ is the injective envelope of ${ }_{R G} R$. Hence by [22] $Q(\widehat{S}) \cong \operatorname{End}_{R}(Q(R))$.

Note that if in addition to the above conditions we assume $R^{G}$ is prime then the context is prime and $\hat{S}$ is prime.

As a corollary to Theorem 1.22 we have:

THEOREM 1.23. Let $R$ be a ring and assume $S$ is semiprime and $R$ is a faithful $S$-module. Then $S$ is Goldie if and only if $R^{G}$ is Goldie if and only if $R$ is Goldie. And then $Q(S)=\operatorname{End}_{R^{G}}(Q(R))$. Furthermore, $R^{G}$ is prime Goldie if and only if $S$ is prime Goldie.

Proof. By Theorem 1.22 $S$ Goldie implies $R^{G}$ Goldie. Now, if $R^{G}$ is Goldie then by Theorem 1.15.f, $d\left(_{R G} R\right)<\infty$, hence by Theorem $1.22 R$ and $S$ are Goldie and $Q(S)=\operatorname{End}_{R^{G}}(Q(R))$. By Theorem 1.6.a $R$ is Goldie if and only if $R^{G}$ is Goldie. Finally, by Theorem 1.16 $R^{G}$ is prime if and only if $S$ is prime.

1E. G-Galois extensions and related aspects. In [2], Auslander and Goldman introduced the notion of a Galois extension of a commutative ring. Chase, Harrison and Rosenberg [5] have given an equivalent definition which was later used by Miyashita [14] for noncommutative rings. Let us define a G-Galois extension. Let $R$ be a ring with $1, G$ a finite group of automorphisms of $R$, then $R$ will be called a $G$-Galois extension of $R^{G}$ with Galois group $G$ if, (1) $R^{G}=t_{G}(R)$, (2) there exist $x_{1}, \cdots, x_{n}$ and $y_{1}, \cdots, y_{n}$ in $R$ such that

$$
\sum_{i=1}^{n} x_{i} y_{i}^{g}= \begin{cases}1 & \text { if } g=1 \\ 0 & \text { if } g \neq 1\end{cases}
$$


(Miyashita dropped (1) from his definition.) Obviously, (1) is equivalent to $($,$) being onto R^{G}$. Condition (2) is equivalent to [ , ] being onto $S$. For if [,] is onto, then $1=\sum_{i=1}^{n}\left[x_{i}, y_{i}\right]$ for some $\left\{x_{i}, y_{i}\right\} \subset R$, but then $1=\Sigma x_{i} t y_{i}=\sum_{i=1}^{n} \sum_{g \in G} x_{i} y_{i}^{g^{-1}} g=\sum_{g \in G}\left(\Sigma x_{i} y_{i}^{g^{-1}}\right) g$, hence (2) is satisfied. The converse is now obvious. Let us recall a version of the Morita theorem [18].

Theorem (Morita). Let $V$ be a left $A$-module and $B=\operatorname{End}_{A}(V)$. Then the following are equivalent:

(1) Some direct sum of copies of ${ }_{A} V$ can be mapped onto $A$ (i.e., $V$ is a generator for ${ }_{A} \mathscr{C l}$ ).

(2) $V_{B}$ is a finitely generated projective $B$-module and $A \cong$ $\operatorname{End}_{B}(V)$.

It is easy to show [ 2 or 18 ] that when $1 \in R$, then $R^{G} \cong \operatorname{End}_{S}(R)$. Furthermore, if [,] is onto $S$ then $1=\sum_{i=1}^{n}\left[w_{i}, v_{i}\right]$. Let $f: \sum_{i=1}^{n} R \rightarrow$ $S$ be defined by: $\sum_{i=1}^{n} r_{i} \rightarrow \sum_{i=1}^{n}\left[r_{i}, v_{i}\right]$. Then $f$ is an $S$-module homomorphism onto $S$. That is, if [, ] is onto $S$, then ${ }_{S} R$ satisfies (1) with $B=R^{G}$. Also, if [,] is onto then it is mono [21, p. 113]. Similar remarks can be made if $($,$) is onto. Finally, define$

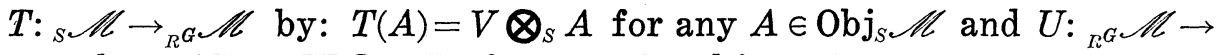
${ }_{s} \mathscr{C l}$ by $U(B)=W \otimes_{R^{G}} B$, for any $B \in \operatorname{obj}_{R} G \mathscr{C l}$.

In the following we use $R$ to denote both $V$ and $W$. It is understood, however, from the context of things, which module structure we mean.

LEMMA 1.24. Let $R$ be a semiprime ring with 1. If [, ] is onto $S$ then:

(a) $R$ is a finitely generated projective $R^{G}$-module.

(b) $R$ is a faithful S-generator.

(c) $S \cong \operatorname{End}_{R^{G}}(R)$ as an $R^{G}$-module.

(d) The mapping [, ]: $R \bigotimes_{R^{G}} R \rightarrow S$ is an S-bimodule isomorphism.

(e) The functor UT is naturally equivalent to the identity functor on $s \mathscr{M}$.

LEMMA $1.24^{\prime}$. Let $R$ be a semiprime ring with 1 and let $B=$ $\operatorname{End}_{R^{G}}(R) . \quad$ If $($,$) is onto R^{G}$ then:

$($ a) $R$ is a finitely generated projective $B$-module.

(b)' $R$ is a faithful $R^{G}$-generator.

(c) $)^{\prime} \quad R^{G} \cong \operatorname{End}_{B}(R)$.

(d)' The mapping (, ): $R \bigotimes_{S} R \rightarrow R^{G}$ is an $R^{G}$ bimodule isomorphism.

$(\mathrm{e})^{\prime}$ The functor $T U$ is naturally equivalent to the identity 
functor on ${ }_{R} \mathscr{C l}^{\mathrm{M}}$.

(f)' $\quad R^{G}$ is a direct summand of ${ }_{R} G R\left(R_{R^{G}}\right)$.

Proof. (a), (b), (c), (d) follow from the remarks and the Morita theorem, (e) follows from (d), (f) follows since $t_{G}$ is onto.

Consequently, a lemma which also appears in [5].

LEMMA 1.25. Let $R$ be a semiprime ring with 1 which is a G-Galois extension of $R^{G}$. Then $R^{G}$ is Morita equivalent to $S$, in particular:

(a) $R$ is finitely generated, faithful and projective both as an $R^{G}$-module and an S-module.

(b) $S \cong \operatorname{End}_{R^{G}}(R)$ and $R^{G} \cong \operatorname{End}_{S}(R) ; \quad S \cong{ }_{S} R \bigotimes_{R^{G}} R$ and $R^{G} \cong$ ${ }_{R}^{G} R \otimes_{S} R$.

Furthermore, UT and TU are naturally equivalent to the identity functor on ${ }_{S} \mathscr{C l}$ and ${ }_{R^{G}} \mathscr{C l}$ respectively.

(c) $R^{G}$ is a direct summand of ${ }_{R^{G}} R\left(R_{R^{G}}\right)$.

Let $\mathscr{L}\left({ }_{n} M\right)$ denote the lattice of $R$-submodules of $M$. We shall briefly outline some connections between $\mathscr{L}\left({ }_{R} G R\right)$ and $\mathscr{L}\left({ }_{S} S\right)$. Let $\sigma: \mathscr{L}\left({ }_{{ }_{R G} G} R\right) \rightarrow \mathscr{L}\left({ }_{S} S\right)$ be defined by $\sigma(A)=[W, A]$ and $\tau: \mathscr{L}\left({ }_{S} S\right) \rightarrow$ $\mathscr{L}\left({ }_{R} G\right)$ be defined by $\tau(X)=V \cdot X$. Now $\tau \sigma(A)=V \cdot[W, A]=(V, W) A$ and $\sigma \tau(X)=[W, V \cdot X]=[W, V] X$. If $1 \in R$ and $($,$) is onto then$ $\tau \sigma=\mathrm{id}_{\left.\left.\mathscr{S}_{(} G^{R}\right)^{R}\right)}$. In particular, $\sigma$ is order preserving. Similarly, if $[$,$] is onto then \tau$ is order preserving hence we have:

THEOREM 1.26. Let $R$ be a semiprime ring with 1 . If $($,$) is$ onto and ${ }_{R} R$ is Noetherian (Artinian), then so is ${ }_{R_{G}} R$.

Proof. If ${ }_{R} R$ is Noetherian (Artinian) then since ${ }_{R} S$ is a finite free $R$-module it is Noetherian (Artinian), but then so is ${ }_{S} S$. The result now follows since $\sigma$ is order preserving.

THEOREM 1.27. Let $R$ be a ring. If $S$ is a simple ring then:

(1) [, ] is onto $S$ and ${ }_{s} W$ is a faithful S-module.

(2) $R^{G}$ is primitive.

(3) If $1 \in S$ then $(V, W)$ is the intersection of all nonzero ideals of $R^{G}$, hence, in particular, it is a simple ring.

Proof. (1) Since $R$ is semiprime, $[W, V] \neq 0$, hence as an ideal of $S[W, V]=S$. By Theorem $1.16{ }_{S} W$ is faithful.

(2) Follows from Theorem 1.18.b.

(3) Let $A$ be an ideal of $R^{a}$, then $A \supset(V, W) A(V, W)=$ $(V, W A(V, W))=(V,[W A, V] \cdot W)$. Since $[W A, V]$ is a nonzero 
ideal of $S$ it equals $S$. Hence $A \supset(V, W)$.

We end with a situation in which $R$ is a $G$-Galois extension of $R^{G}$.

THEOREM 1.28. Let $R$ be a semisimple Artinian ring and a faithful $S$-module and assume $R^{G}$ (or $S$ ) is a semiprime ring then $R$ is a G-Galois extension of $R^{G}$, and ${ }_{R^{G}} R$ is Artinian.

Proof. Since ${ }_{R} S$ is a finite free $R$-module, ${ }_{R} S$ hence ${ }_{S} S$ is Artinian. By Theorem 1.18.a $S$ is semiprime, hence semisimple Artinian. By Theorem 1.15, $[W, V]$ is an essential ideal of $S$, thus $[W, V]=S$. That is, [,] is onto. By Theorem 1.15.b (, ) is nondegenerate hence by Theorem 1.6.b ( , ) is onto. We have shown that $R$ is a $G$-Galois extension of $R^{G},{ }_{R^{G}} R$ is Artinian by Theorem 1.26 .

2. Applications. We shall apply results of $\S 1$ to two situations:

(1) $R$ is semiprime and $G$ is a finite group of $X$-outer automorphisms.

(2) $R$ is a semiprime, $|G|$-torsion free ring, where $G$ is a finite group $\subset \operatorname{Aut}(R)$.

As mentioned in the introduction, in both cases $S$ is semiprime, and in (1) $R$ is also $S$-faithful. The following example suggested by Montgomery shows that this need not be true in case (2).

EXAMPLE 2.1. Let $R=M_{3}(Q)$, where $Q=$ rationals. Let $x=$ $\left(\begin{array}{rrr}1 & 0 & 0 \\ 0 & 1 & 0 \\ 0 & 0 & -1\end{array}\right), y=\left(\begin{array}{rrr}1 & 0 & 0 \\ 0 & -1 & 0 \\ 0 & 0 & 1\end{array}\right)$ and let $g, h, g h$ denote the inner automorphisms of $R$ determined by $x, y$ and $x y$ respectively. Then $G=\langle 1, g, h, g h\rangle$ is a (abelian) group of inner automorphisms of $R$. However $x+y-$ $(x y+1)=0$, hence $R \cdot[x g-y h-x y g h+1]=0$. Thus $R$ is not a faithful $S$-module. Note that, as expected, $\operatorname{dim}_{C} B=3<\left|G_{\text {inn }}\right|$.

Let us deal first with (1). Some of the results appeared in the work of $[11,14,15,18,19]$.

THEOREM 2.2. Let $R$ be a semiprime ring and $G$ be a finite group of $X$-outer automorphisms. Then:

(a) $(V, W)$ is an essential ideal of $R^{a}$.

(b) $[W, V],[W, V] \cap R$ and $[W, V] \cap R^{G}$ are essential ideals of $S, R$ and $R^{G}$ respectively.

(c) $R^{G}$ is a semiprime ring.

(d) (, ) and [, ] are nondegenerate.

(e) $d\left({ }_{R^{G}} R\right)=d\left({ }_{S} S\right) \leqq|G|^{2} d\left({ }_{{ }_{R} G} R^{G}\right)$. 
Proof. (a), (d) and (e) follow from Theorem 1.15. (c) $S$ is semiprime, hence by Theorem $1.21, R^{G}$ is semiprime. (b) $[W, V]$ is essential in $S$ by Theorem 1.15. Now let $B=[W, V] \cap R$, and $A=$ $A_{n}(B)$. Then $[A W, V]$ is an ideal of $S$. However, $([A W, V] \cap$ $R)^{2} \subset([A W, V] \cap R)[A W, V]=([A W, V] \cap R) A[W, V]=0$. Thus by Theorem $0.1[A W, V]=0$ which by nondegeneracy implies $A=0$. Hence $B$ is essential in $R$. Now by Lemma $1.3(B, W)$ is essential in $R^{G}$. However, since $B$ is $G$-invariant $(B, W) \subset B \cap R^{G}=[W, V] \cap$ $R^{G}$.

Next we impose various conditions on the rings, (b) extends a result of [11], (c) gives an affirmative answer to Question 11 of [10]. (d) is partially proved in [15].

THEOREM 2.3. Let $R$ be a semiprime ring and $G$ a finite group of $X$-outer automorphisms, then:

(a) $\mathscr{N}\left(R^{G}\right)=0$ if and only if $\mathscr{N}(S)=0$ where $\mathscr{N}\left({ }^{*}\right)$ is one of the: Jacobson, locally nilpotent or nil radicals. Thus $\mathscr{N}(R)=0$ implies $\mathscr{N}\left(R^{G}\right)=0$.

(b) $R$ is G-prime if and only if $S$ is prime if and only if $R^{G}$ is prime.

(c) $S$ is primitive if and only if $R^{G}$ is primitive. Thus if $R$ is primitive then so is $R^{G}$.

(d) $S$ is Goldie if and only if $R^{G}$ is Goldie if and only if $R$ is Goldie, and then $Q(S)=\operatorname{End}_{R^{G}}(Q(R))$.

(e) If $R^{G}$ is an Ore domain then $S$ is a prime Goldie ring, $Q(S)=\left(Q\left(R^{G}\right)\right)_{n}$ where $n=d\left({ }_{S} S\right)=d\left(_{R^{G}} R\right) \leqq|G|^{2}$.

Proof. (a) The first part follows from Theorem 1.18. Now if $\mathscr{N}(R)=0$ then since $\mathscr{N}(S) \cap R \subset \mathscr{N}(R)=0$, Theorem 0.1 implies that $\mathscr{N}(S)=0$, which by the first part implies that $\mathscr{N}\left(R^{G}\right)=0$.

(b) It is easy to see that $R$ is $G$ prime if and only if $S$ is prime [13]. The rest follows from Theorem 1.16.

(c) The first part follows from Theorem 1.18. Now, if $R$ is primitive then so is $S$ [8], hence so is $R^{a}$.

(d) and (e) follows from Theorems 1.23 and 1.19 respectively.

We shall next give instances in which $R$ is $G$-Galois over $R^{G}$. The following generalizes a result of [3] from simple Artinian to semisimple Artinian rings.

THEOREM 2.4. Let $R$ be a semisimple Artinian ring and assume $G$ is a finite group of $X$-outer automorphisms then $R$ is a G-Galois extension of $R^{G}$. In particular, $R^{G}$ and $S$ are Morita equivalent hence semisimple Artinian, and $S=\operatorname{End}_{R^{G}} R . A l s o, R$ is finitely generated, projective and faithful both as an $R^{G}$ and an S-module, 
and ${ }_{R^{G}} R$ is Artinian.

Proof. By Theorem 1.28 and Lemma 1.25.

THEOREM 2.5. Let $R$ be a semiprime ring with 1 and $G$ a finite group of $X$-outer automorphisms. If $R^{G}$ is a simple ring then $R$ is a G-Galois extension of $R^{G}$.

Proof. By simplicity of $R^{G},($,$) is onto. Now by Theorem$ 2.2.b $[W, V] \cap R^{G} \neq 0$ is an ideal of $R^{G}$ hence $1 \in[W, V]$ implying [ , ] is onto. Thus, $R$ is a G-Galois extension of $R^{G}$.

We next record results of $[14,19,20]$ which were proved using ideas similar to the ones in $\S 1$.

THEOREM 2.6. Let $R$ be a simple ring and assume $G$ is a finite group of $X$-outer automorphisms of $R$. Then

(a) $[$,$] is onto.$

(b) $R^{G}$ is a primitive ring.

If in addition, $1 \in R$ then:

(c) $R$ is a finitely generated projective $R^{G}$-module.

(d) $S \cong \operatorname{End}_{R^{G}}(R)$ and $R$ is a faithful $S$-generator.

(e) $(V, W)$ is a simple ring and is the intersection of all nonzero ideals of $R^{G}$.

Proof. $S$ is simple by [8] hence the result follows from Lemma 1.24 and Theorem 1.27.

We conclude by considering case (2).

THEOREM 2.7. Let $G$ be a finite group of automorphisms of a semiprime, $|G|$-torsion free ring then:

(a) $R^{G}$ is semiprime.

(b) $(V, W)$ is an essential ideal of $R^{a}$.

(c) (, ) is nondegenerate.

(d) $R^{G}$ is Goldie if and only if $R$ is Goldie and then $Q\left({ }_{R} R\right)=$ $Q\left({ }_{R G} R\right)$.

(e) $R^{G}$ is Artinian if and only if $R$ is Artinian and then (, ) is onto. Hence, in particular $R \otimes_{S} R \cong R^{G}$ (as an $R^{G}$-bimodule), ${ }_{R_{G}^{G}} R$ is Artinian and $R^{G}$ is a direct summand of ${ }_{R^{G}} R$.

(f) $\hat{S}=S / \mathrm{Ann}_{S} R$ is Goldie if and only if $d\left(_{R_{G}} R\right)<\infty$ and $Z\left({ }_{R_{G}} R^{G}\right)=0$, and then $R$ and $R^{G}$ are semiprime Goldie and $Q(\hat{S}) \cong$ $\operatorname{End}_{R G}(Q(R))$.

Proof. (a), (b), (c), (d) follow from Theorems 1.21 and 1.26, (f) follows from Theorem 1.22. 
Added in proof. Theorem 2.3.c has been proved independently by J. L. Pascaud in: Two results on fixed rings.

\section{REFERENCES}

1. S. A. Amitsur, Rings of quotients and Morita contexts, J. Algebra, 17 (1971), 273-298.

2. M. Auslander and O. Goldman, The Brauer group of a commutative ring, Trans. Amer. Math. Soc., 97 (1960), 367-409.

3. G. Azumaya and T. Nakayama, On irreducible rings, Annals of Math., (1947), 949-965.

4. G. M. Bergman and I. M. Isaacs, Rings with fixed point free group actions, Proc. London Math. Soc., 27 (1973), 69-87.

5. S. U. Chase, D. K. Harrison and A. Rosenberg, Galois theory and cohomology of commutative rings, Mem. Amer. Math. Soc., 52 (1965).

6. M. Cohen, Semiprime Goldie centralizers, Israel J. of Math., 20 (1975), 37-45.

7. M. Cohen and S. Montgomery, Trace functions for finite automorphism groups of rings, Math. Arch., (to appear).

8. J. Fisher and S. Montgomery, Semiprime skew group rings, J. Algebra, 52 (1978), 241-247.

9. J. Fisher and J. Osterberg, Semiprime ideals in rings with finite group actions, J. Algebra, 50 (1978), 488-502.

10. - Finite group actions on noncommutative rings: a survey since 1970, Proc. of 1979 Oklahoma Conf., (to appear).

11. V. K. Kharchenko, Generalized identities with automorphisms, Algebra and Logic (trans.) (1976), 132-148.

12. — Fixed elements under a group acting on a semiprime ring, Algebra and Logic (trans.) (1976), 203-213.

13. M. Lorentz and D. S. Passman, Prime ideals in crossed products of finite groups, Israel J. Math., 33 (1979), 89-132.

14. Y. Miyashita, Finite outer Galois theory of noncommutative rings, J. Fac. Sci. Hokkaido Univ., 19 (1966), 114-134.

15. S. Montgomery, Outer automorphisms of semiprime rings, J. London Math. Soc., 18 (1978), 209-221.

16. - Automorphism groups of rings with no nilpotent elements, J. Algebra, 60 (1979).

17. S. Montgomery and D. S. Passman, Crossed products over prime rings, Israel J. Math., 31 (1978), 224-256.

18. - Fixed rings of finite automorphism groups of associative rings, Lecture Notes in Math., Springer-Verlag, 1980, (to appear).

19. O. M. Neroslavskii and A. E. Zaleskii, On simple Noetherian rings, Proc. Akad. Bylorussia S.S.R., (1975), 38-42.

20. J. Osterburg, Fixed rings of simple rings, Comm. in Algebra, 6 (1978), 1741-1750.

21. B. Stenström, Rings of Quotients, Springer-Verlag, 1975.

22. J. Zelmanowitz, Semiprime modules with maximum conditions, J. Algebra, 25 (1973), 554-574.

Received September 18, 1980 and in revised form February 27, 1981.

Ben Gurion University of The Negev

Beer Sheva, IsRael 


\title{
PACIFIC JOURNAL OF MATHEMATICS
}

\section{EDITORS}

\author{
DONALD BABBITT (Managing Editor) \\ University of California \\ Los Angeles, CA 90024 \\ Hugo RossI \\ University of Utah \\ Salt Lake City, UT 84112 \\ C. C. Moore and ANDrew OGG \\ University of California \\ Berkeley, CA 94720
}

\section{J. DugundjI}

Department of Mathematics

University of Southern California

Los Angeles, CA 90007

R. FinN and J. Milgram

Stanford University

Stanford, CA 94305

\section{ASSOCIATE EDITORS}
R. ARENS
E. F. BeCKENBACH
B. H. NeUMANN
F. WOLF
K. YoshidA

\section{SUPPORTING INSTITUTIONS}

UNIVERSITY OF ARIZONA

UNIVERSITY OF BRITISH COLUMBIA

CALIFORNIA INSTITUTE OF TECHNOLOGY

UNIVERSITY OF CALIFORNIA

MONTANA STATE UNIVERSITY

UNIVERSITY OF NEVADA, RENO

NEW MEXICO STATE UNIVERSITY

OREGON STATE UNIVERSITY

\author{
UNIVERSITY OF OREGON \\ UNIVERSITY OF SOUTHERN CALIFORNIA \\ STANFORD UNIVERSITY \\ UNIVERSITY OF HAWAII \\ UNIVERSITY OF TOKYO \\ UNIVERSITY OF UTAH \\ WASHINGTON STATE UNIVERSITY \\ UNIVERSITY OF WASHINGTON
}

The Supporting Institutions listed above contribute to the cost of publication of this Journal, but they are not owners or publishers and have no responsibility for its content or policies.

Mathematical papers intended for publication in the Pacific Journal of Mathematics should be in typed form or offset-reproduced, (not dittoed), double spaced with large margins. Please do not use built up fractions in the text of the manuscript. However, you may use them in the displayed equations. Underline Greek letters in red, German in green, and script in blue. The first paragraph or two must be capable of being used separately as a synopsis of the entire paper. Please propose a heading for the odd numbered pages of less than 35 characters. Manuscripts, in triplicate, may be sent to any one of the editors. Please classify according to the scheme of Math. Reviews, Index to Vol. 39. Supply name and address of author to whom proofs should be sent. All other communications should be addressed to the managing editor, or Elaine Barth, University of California, Los Angeles, California, 90024.

50 reprints to each author are provided free for each article, only if page charges have been substantially paid. Additional copies may be obtained at cost in multiples of 50 .

The Pacific Journal of Mathematics is issued monthly as of January 1966. Regular subscription rate: $\$ 102.00$ a year (6 Vols., 12 issues). Special rate: $\$ 51.00$ a year to individual members of supporting institutions.

Subscriptions, orders for numbers issued in the last three calendar years, and changes of address shoud be sent to Pacific Journal of Mathematics, P.O. Box 969, Carmel Valley, CA 93924, U.S.A. Old back numbers obtainable from Kraus Periodicals Co., Route 100, Millwood, NY 10546.

PUBLISHED BY PACIFIC JOURNAL OF MATHEMATICS, A NON-PROFIT CORPORATION

Printed at Kokusai Bunken Insatsusha (International Academic Printing Co., Ltd.). 8-8, 3-chome, Takadanobaba, Shinjuku-ku, Tokyo 160, Japan. 


\section{Pacific Journal of Mathematics}

\section{Vol. 98, No. 1 \\ March, 1982}

Humberto Raul Alagia, Cartan subalgebras of Banach-Lie algebras of

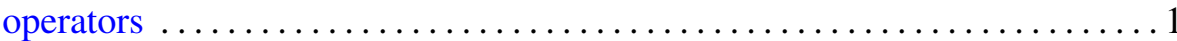

Tom M. (Mike) Apostol and Thiennu H. Vu, Elementary proofs of

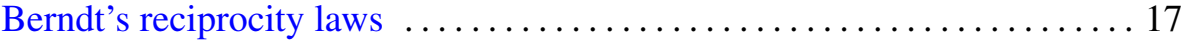

James Robert Boone, A note on linearly ordered net spaces $\ldots \ldots \ldots \ldots . \ldots 25$

Miriam Cohen, A Morita context related to finite automorphism groups of

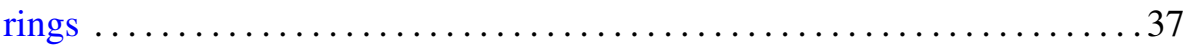

Willibald Doeringer, Exceptional values of differential polynomials . . . . . . 55

Alan Stewart Dow and Ortwin Joachim Martin Forster, Absolute

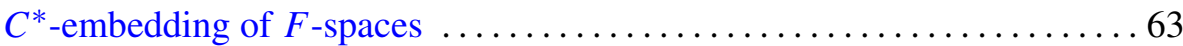

Patrick Hudson Flinn, A characterization of $M$-ideals in $B\left(l_{p}\right)$ for

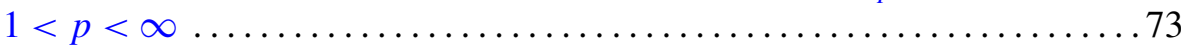

Jack Emile Girolo, Approximating compact sets in normed linear spaces . . . 81

Antonio Granata, A geometric characterization of $n$th order convex

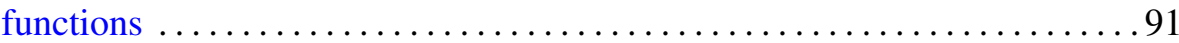

Kenneth Richard Johnson, A reciprocity law for Ramanujan sums . . . . . . .99

Grigori Abramovich Kolesnik, On the order of $\zeta\left(\frac{1}{2}+i t\right)$ and $\Delta(R) \ldots \ldots 107$

Daniel Joseph Madden and William Yslas Vélez, Polynomials that

represent quadratic residues at primitive roots $\ldots \ldots \ldots \ldots \ldots \ldots \ldots 123$

Ernest A. Michael, On maps related to $\sigma$-locally finite and $\sigma$-discrete

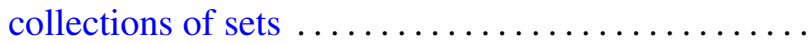

Jean-Pierre Rosay, Un exemple d'ouvert borné de $\mathbf{C}^{3}$ “taut" mais non

hyperbolique complet

Roger Sherwood Schlafly, Universal connections: the local problem

Russel A. Smucker, Quasidiagonal weighted shifts .....

Eduardo Daniel Sontag, Remarks on piecewise-linear algebra . .

Jan Søreng, Symmetric shift registers. II

H. M. (Hari Mohan) Srivastava, Some biorthogonal polynomials suggested

by the Laguerre polynomials 